\title{
A study to improve the past orbit of comet C/1917 F1 (Mellish) on the basis of its observed meteor showers
}

\author{
L. Neslušan ${ }^{1}$, J. Vaubaillon ${ }^{2}$, and M. Hajduková, $\mathrm{Jr}^{3}$ \\ 1 Astronomical Institute, Slovak Academy of Science, 05960 Tatranská Lomnica, Slovakia \\ e-mail: ne@ta3.sk \\ 2 IMCCE, 77 Av. Denfert Rochereau, 75014 Paris, France \\ e-mail: Jeremie.Vaubaillon@obspm. fr \\ 3 Astronomical Institute, Slovak Academy of Science, Dúbravská cesta 9, 84504 Bratislava, Slovakia \\ e-mail: astromia@savba.sk
}

Received 8 October 2015 / Accepted 8 February 2016

\section{ABSTRACT}

\begin{abstract}
Context. Periodic comets are known to be the parent bodies of meteoroid streams. The stream of a given comet can split into several filaments. These can be observed in the Earth's atmosphere as more than just a single meteor shower. One such comet is C/1917 F1 (Mellish), which associates at least two, possibly four, meteor showers that have been recorded in the meteor databases. In a recent study, the dynamical evolution of the C/1917 F1's theoretical stream was followed by only considering the gravitational perturbations. The properties of individual filaments of this stream, corresponding to the appropriate meteor showers, were not predicted perfectly. Aims. To reach better agreement between theory and observation, we repeatedly model the theoretical stream of C/1917 F1. In the modeling, we also include the Poynting-Robertson drag acting on meteoroids and assume an action of the non-gravitational effects on the parent comet dynamics. If success was achieved, the modeling could become a tool that would enable us to recover the past orbital history of the parent comet.

Methods. Considering the nominal orbit, as well as several cloned orbits, of the comet C/1917 F1, we modeled its theoretical streams. The modeling was performed for several past perihelion passages. Each modeled stream consists of 10000 test particles that are influenced by the Poynting-Robertson drag of various strengths.

Results. We achieve a partial improvement in the prediction of the properties of all four meteor showers. The Poynting-Robertson drag helps to improve the match between the theory and observation of three of the four showers. However, when considering the nominal orbit of the parent comet, a perfect match seems to be impossible. A close match in the case of the most problematic shower is achieved using a cloned orbit, but this is not applicable to reality because the simultaneous predictions of the properties of the other three showers fail.
\end{abstract}

Key words. comets: individual: C/1917 F1 (Mellish) - meteorites, meteors, meteoroids

\section{Introduction}

The meteoroid stream of periodic comet C/1917 F1 (Mellish) was recently investigated in the paper by Neslušan \& Hajduková (2014; NH14, hereinafter). It was found that the stream of this comet crosses the Earth's orbit in four filaments, which were denoted (and this denotation is also used in the current paper) as F1, F2, F3, and F4. As a result, four meteor showers associated with $\mathrm{C} / 1917 \mathrm{~F} 1$ were predicted. One of these showers, the December Monocerotids (No. 19 in the IAU MDC list of established showers; Jopek \& Kaňuchová 2014) ${ }^{1}$, corresponding to F3, clearly exists. Its relationship to the comet C/1917 F1 has been confirmed, before NH14, by several authors (e.g., McCrosky \& Posen 1961; Lindblad 1971; Kresáková 1974; Drummond 1981; Olsson-Steel 1987).

Identification of another shower, April $\rho$-Cygnids (No. 348), with the corresponding predicted filament F1, is not very obvious. However, there is at least a strong suspicion that a reliable identification will be possible after a larger set of data becomes available. NH14 used, in general, the photographic IAU MDC (Neslušan et al. 2014), SonotaCo video (SonotaCo 2009), and radio-meteor (Hawkins 1963; Sekanina \& Southworth 1975; Lindblad 2003, priv. comm.) databases in

\footnotetext{
1 https://www.ta3.sk/IAUC22DB/MDC2007/Roje/roje_lista. php?corobic_roje $=1 \&$ sort_roje $=0$
}

their shower identification. With the angular distance of mean radiant from the Sun in the activity maximum lower than $70^{\circ}$, $\rho$-Cygnids were only, however, found in the video data. A similar, non-definitive conclusion about the April $\rho$-Cygnids, selected from several catalogs, and their relationship to C/1917 F1 was also made by Hajduková et al. (2015).

Of another two predicted showers, one (F2) is a daytime shower, which seems to be present in the SonotaCo video and radio-meteor databases. Currently, no corresponding shower can be found in the IAU MDC list of all showers. In the SonotaCo video data, there is also an indication of a shower that resembles the last predicted filament, F4.

Not only the unclear identifications of filaments F1, F2, and F4, but also an imperfect match between the predicted filament F3 and the December Monocerotids demand improvement in any modeling of the creation and subsequent dynamical evolution of the C/1917 F1's stream. In NH14, the influence of the Poynting-Robertson (P-R) drag was not considered in the dynamics of modeled stream particles, so we consider its influence in this work.

As we show in the following, the P-R drag alone does not, however, yield a perfect match, either. In the first part of our modeling, we assumed the past evolution of the nominal osculation orbit of C/1917 F1 only because of the gravitational perturbations of planets. We note that this orbit was 
determined by Asklöf (1932). It is known with relatively high precision. Asklöf gave three sets of orbital elements determined for the different assumptions and selections of observational data. The largest differences of corresponding values in these three sets are only $0.001627 \mathrm{~d}$ in the time of perihelion passage, $14.9^{\prime \prime}$ in the argument of perihelion, 21.2" in longitude of ascending node, and $5^{\prime \prime}$ in the inclination. The orbital elements for the equinox 2000.0, given on the JPL-browser ${ }^{2}$ are $q=0.190186 \mathrm{AU}, e=0.993121, \omega=121.3190^{\circ}, \Omega=88.6683^{\circ}$, $i=32.6828^{\circ}$, and $T=2421329.6751$ (April 11.1751, 1917) for epoch JDT $=2421333.5$.

In the past, this orbit could be significantly influenced and, therefore, modified by non-gravitational effects. The currently observed stream could be and, most likely, was formed when the comet moved in a slightly different orbit than it moved at its last return to the perihelion.

To model a theoretical stream and follow its dynamical evolution, we use the same procedure as Neslušan and Hajduková in NH14 (see Sect. 2 in their paper). The procedure starts with the integration of the parent-body orbit backward for time $t_{\mathrm{ev}}$. Then, the nearest perihelion is found and a set of 10000 test particles (TPs), representing the meteoroids, are assumed to be ejected from the parent nucleus with a small velocity in all directions. Finally, the orbits of the TPs are integrated forward, up to the present. The TPs in the orbits that approach the Earth's orbit within $0.05 \mathrm{AU}$ at the present time are selected and used to predict the properties of relevant showers.

Our theoretical predictions are evaluated by comparing them to the actually observed meteors. In this comparison, we use the photographic IAU MDC (Neslušan et al. 2014), SonotaCo video (SonotaCo 2009), and radio-meteor (Hawkins 1963; Sekanina \& Southworth 1975; Lindblad 2003, priv. comm.) databases. In contrast to NH14, we also use the EDMOND video database (Kornoš et al. 2014).

In this research note, we describe our attempt to find the appropriate strength of the P-R drag influencing the dynamical evolution of the C/1917 F1 stream and causing four meteor showers in the Earth's atmosphere. The strength of the drag is given with the help of parameter $\beta$, which is the ratio of the acceleration due to the P-R drag and gravitational acceleration of the Sun. Likewise, because of non-gravitational effects in general, we try to find the appropriate modification of the orbit of this parent body in the time when the meteoroids of its stream were released. A more detailed description of the problem solved is given in the next section.

This work aims not only to better predict the properties of the showers associated with the parent comet, but also to point out the possibility of an eventual recovery of the past orbital history of the comet by studying its observed meteor showers. If our work or other future work of this kind is successful, then the meteor databases will become a tool for tracing the past dynamical history (caused by the non-gravitational effects) of those parent bodies that associate more than a single meteor shower.

\section{Description of problem}

We first recall the differences between the predicted and observed filaments of the meteoroid stream of comet C/1917 F1 (Mellish), which have already been found in NH14. In their work, the authors exclusively considered the gravitational perturbations of planets on the meteoroid particles.

\footnotetext{
2 http://ssd.jpl.nasa.gov/sbdb.cgi
}

These differences are discussed in terms of the positions of the radiant areas of individual filaments from F1 to F4. We note that the members of all four filaments were selected from the observational databases considered, using the "break-point method" (Neslušan et al. 1995, 2013).

Filaments F1, F2, and F4 can be separated well only in the SonotaCo catalog, and filament F2 also seems to be present in the radio data. The break point is not very apparent when the EDMOND video data are used to separate F1, F2, and F4. Concerning the F3 filament, very similar mean shower characteristics are obtained from both the SonotaCo and EDMOND catalogs, and this rich filament is present in the photographic IAU MDC and radio-meteor data, of course.

The positions of the observed, as well as the predicted, radiants are shown in Fig. 1 for the model with the evolutionary time $t_{\mathrm{ev}}=50 \mathrm{kyr}$. From the observed meteors, the photographic and SonotaCo video meteors are shown. Since the radiant area of filament F3 separated from the EDMOND catalog is almost identical to the area separated from the SonotaCo database, the EDMOND video meteors are not plotted in Fig. 1 or in the other figures. The predicted radiant positions represent all TPs approaching the Earth's orbit within 0.05 AU at present.

We can see that the radiant area of filament F1, which can hypothetically be identified to the April $\rho$-Cygnids (No. 348) as suggested in NH14, is almost predicted correctly. (There is, nevertheless, a "tail" of radiants in the southeast direction.) The radiant area of filament F2 is shifted toward a larger right ascension and, significantly, toward a higher declination. In addition, in filament F4, a low abundance of meteors is predicted, which does not match the observed radiant area perfectly. The predicted radiant area of filament F3, identified with the December Monocerotids (No. 19), more or less matches its video observed counterpart, although it is shifted slightly southward.

The differences between the prediction and observation described above could occur because of the non-gravitational effects, which were not taken into account in NH14. The most relevant non-gravitational effects are the P-R drag and the rocket effect on the nucleus of the parent comet. The first effect influences the orbits of meteoroids. The second changes the orbit of the parent; therefore, the particles could be released from the parent's nucleus in a different position and/or a different time than implied by the well-known nominal orbit of the C/1917 F1. In the following, we trace the action of these effects in the past.

\section{Nominal orbit and P-R drag}

\subsection{An initial discussion}

The orbital period of C/1917 F1 is about 145 years, so its stream has needed a relatively long time to spread along the whole orbit. This and the relatively short perihelion distance, 0.19 AU, of its orbit imply that it is reasonable to also consider the influence of solar radiation and solar wind on the dynamics of especially smaller particles in the meteoroid stream of this comet. Therefore, we add the acceleration due to the P-R drag to the gravitational acceleration of the TPs. To see the influence of this non-gravitational force, we constructed the stream models mostly for $t_{\mathrm{ev}}=5,10,20$, and $50 \mathrm{kyr}$ and followed the dynamics of several streams for each $t_{\mathrm{ev}}$, with different values of parameter $\beta$, which characterizes the strength of the P-R drag.

In the following, we give a description of the achieved results based on a comparison of predicted and observed radiant areas of individual filaments. The results were obtained after modeling theoretical streams, starting from the nominal 


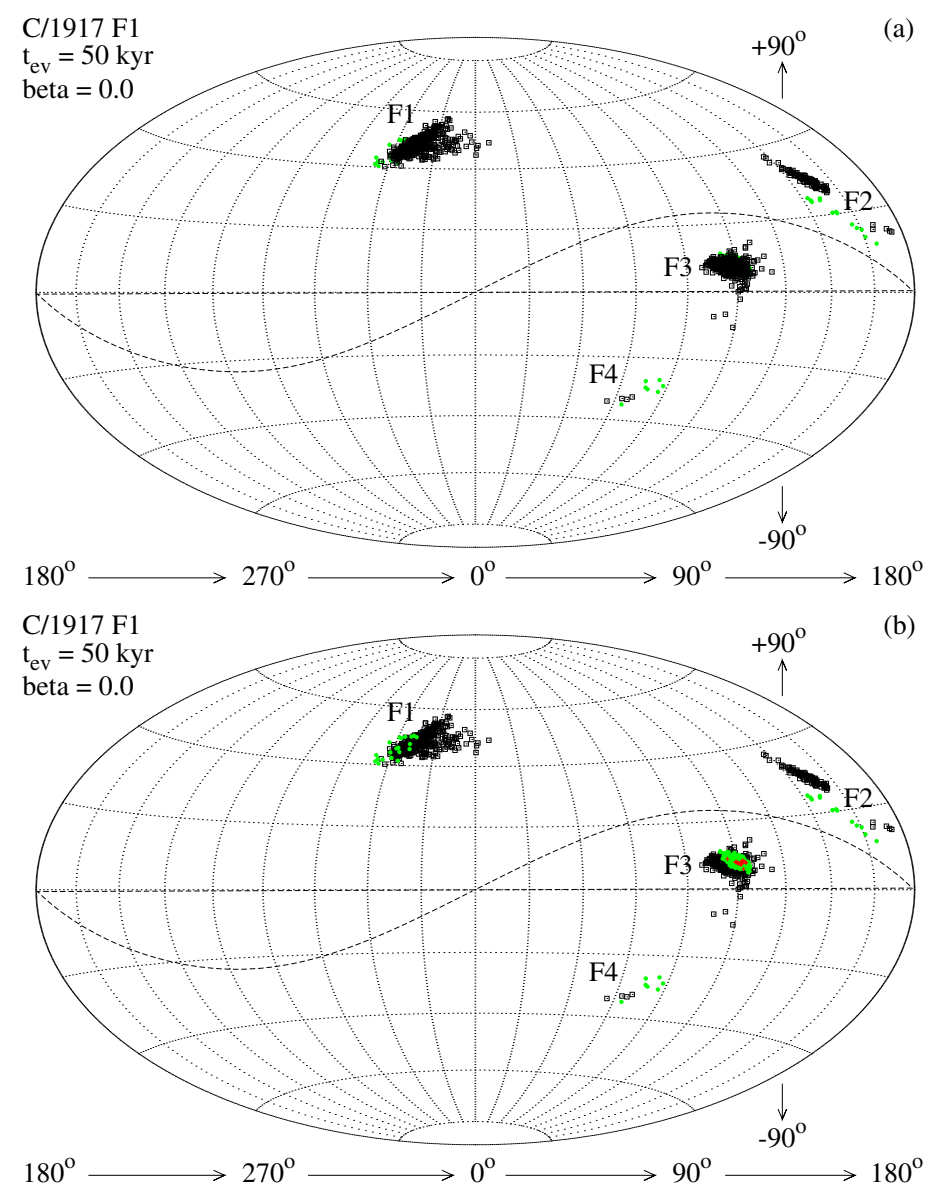

Fig. 1. Positions of radiants of the particles representing the modeled stream of comet C/1917 F1 in orbits, along which they approach the Earth's orbit within $0.05 \mathrm{AU}$. The positions are shown for the stream not influenced by the P-R drag, which was modeled in time 50 kyr in the past. The Hammer projection of the equatorial coordinate frame is used. The green full circles (red crosses) show the positions of the radiants of observed meteors recorded in the SonotaCo-video (photographic IAU MDC) database. The black empty squares show the positions of the radiants of modeled particles. The sinusoid-like curve illustrates the ecliptic. The positions are shown in two variants. In plot a), the radiants of observed meteors are covered by those of modeled particles; therefore, the latter are seen transparently. In contrast, the radiants of modeled particles are covered by those of observed meteors in plot $\mathbf{b}$ ). The positions of the radiants of observed meteors are seen transparently here. The positions of observed radiants are identical in all presented figures.

orbit of $\mathrm{C} / 1917 \mathrm{~F} 1$, and for various values of P-R drag parameter $\beta$. Specifically, the radial, $a_{\mathrm{r}}$, and transversal, $a_{\mathrm{t}}$, components of the acceleration due to the drag were calculated as $a_{\mathrm{r}}=\beta\left(G M_{\odot} / r^{2}\right)\left(1-2 v_{\mathrm{r}} / c\right)$ and $a_{\mathrm{t}}=-\beta\left(G M_{\odot} / r^{2}\right)\left(v_{\mathrm{t}} / c\right)$, where $G$ is the gravitational constant, $r$ the heliocentric distance of TP, $v_{\mathrm{r}}$ and $v_{\mathrm{t}}$ are the radial and transversal component of the TP velocity vector, and $c$ is the speed of light. The transversal component of the acceleration is perpendicular to the radial component and lies in the actual orbital plane of the TP. It is oriented in the direction of the TP velocity. The third component of the P-R-drag acceleration, which is perpendicular to both radial and transversal components, is considered to be zero.

\subsection{An influence of different evolutionary times}

The distributions of the current TP radiants in the streams modeled from the nominal orbit of C/1917 F1 integrated backward to times of $5,10,20,30,50$, and $70 \mathrm{kyr}$ before the present, for $\beta=0.001$, are shown in Fig. 2. The radiants of corresponding shower meteors are also shown in this and in all the other figures for a comparison. This investigation of the influence of evolutionary period on the structure of stream confirms the expected behavior. After the short periods, $t_{\mathrm{ev}}=5$ and $10 \mathrm{kyr}$, only filament F3, which is situated in an orbital corridor around the orbit of the parent comet, appears.

Increasing evolutionary time and thus a longer action of some systematic planetary perturbations enables moving a significant part of the TPs also into other orbital corridors. For $t_{\mathrm{ev}}=20 \mathrm{kyr}$, filaments F1 and F2 also occur. Modeled filament F2 is, however, far from its observed counterpart. The modeled radiant area tends to agree more with that of observed radiants with the increasing $t_{\mathrm{ev}}$. However, F2 starts to disappear in $t_{\mathrm{ev}}=70 \mathrm{kyr}$ when the best match between the radiants of modeled and observed meteoroids is reached. In addition, modeled filaments F1 and F3 become overabundant for the long evolutionary times, $50 \mathrm{kyr}$ and $70 \mathrm{kyr}$.

\subsection{An influence of different strengths of $P-R$ drag}

If we fix the evolutionary period, e.g. $t_{\mathrm{ev}}=50 \mathrm{kyr}$, and consider various values of parameter $\beta$, we can map the influence of the strength of the P-R drag on the evolution of stream structure. The distributions of radiants in this case are presented in Fig. 3. Specifically, we show there the radiant distributions for $\beta$ equal to $0.0001,0.001,0.002,0.003,0.005$, and 0.007 . 


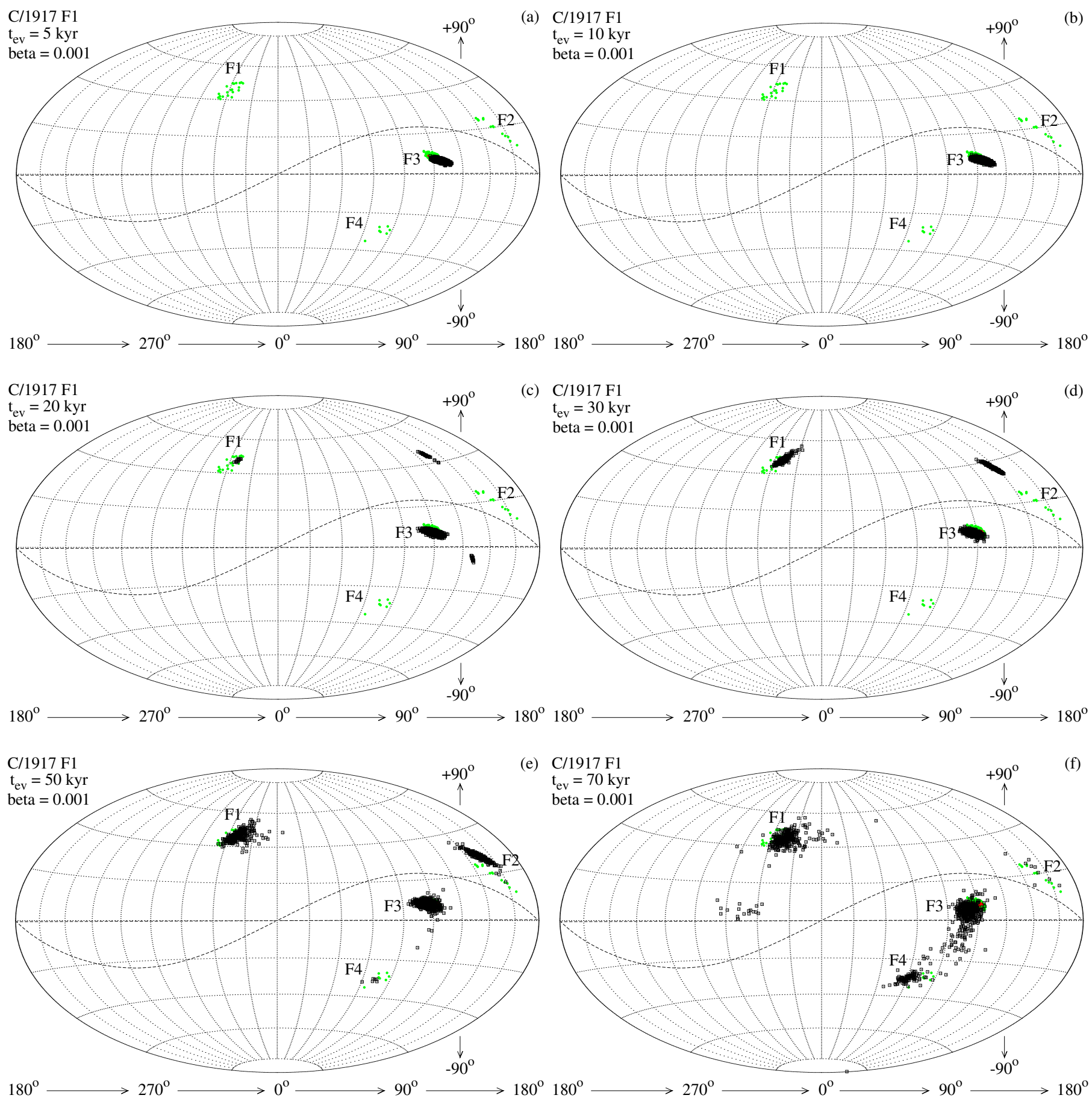

Fig. 2. Positions of radiants of the modeled particles for different evolutionary times. The positions of radiants are shown in the same way as those in Fig. 1a. The P-R drag influenced the motion of modeled particles in this case. The strength of the drag characterized by parameter $\beta$ was 0.001 . The streams with the radiants in plots a)-f) were modeled for times 5, 10, 20, 30, 50, and $70 \mathrm{kyr}$ in the past, respectively.

The dependence on the value of $\beta$ is weaker than on $t_{\mathrm{ev}}$. One can "tune" the position of radiant areas of the filaments and their relative abundances. Nevertheless, a simultaneous perfect match of all mutually corresponding theoretical and observed filaments hardly appears to be achieved. The best match is seen for $\beta$ ranging from $\sim 0.001$ to $\sim 0.003$. Unfortunately, the radiant areas of modeled filaments F2 and F4 are still discernibly displaced from their observed counterparts, and modeled filaments F1 and F3 are much more abundant than observed.

A more detailed description of each modeled stream is given in the following sections.

\subsection{Streams modeled $5 \mathrm{kyr}$ before the present}

In the stream modeled for $t_{\mathrm{ev}}=5 \mathrm{kyr}$, only the TPs in the F3 filament approach the Earth's orbit. In more detail, the F3 filament occurred for $\beta=0.001$ (Fig. 2a) and 0.002 (not shown in a figure). For significantly higher $\beta$ values, all TPs are deflected from the orbit of our planet and, therefore, from the collision course.

\subsection{Streams modeled $10 \mathrm{kyr}$ before the present}

Almost the same result is obtained for $t_{\mathrm{ev}}=10 \mathrm{kyr}$ : only the F3 filament occurs for $\beta=0.001$ (Fig. 2b) and 0.002. For $\beta=0.003$, 
C/1917 F1

$\mathrm{t}_{\mathrm{ev}}=50 \mathrm{kyr}$

beta $=0.000$

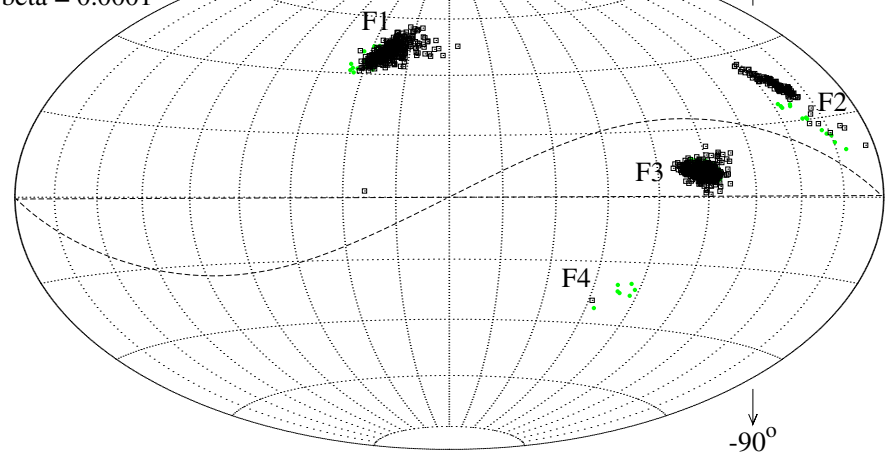

$180^{\circ}$ $270^{\circ}$ $\rightarrow 0^{\circ}$ $90^{\circ}$

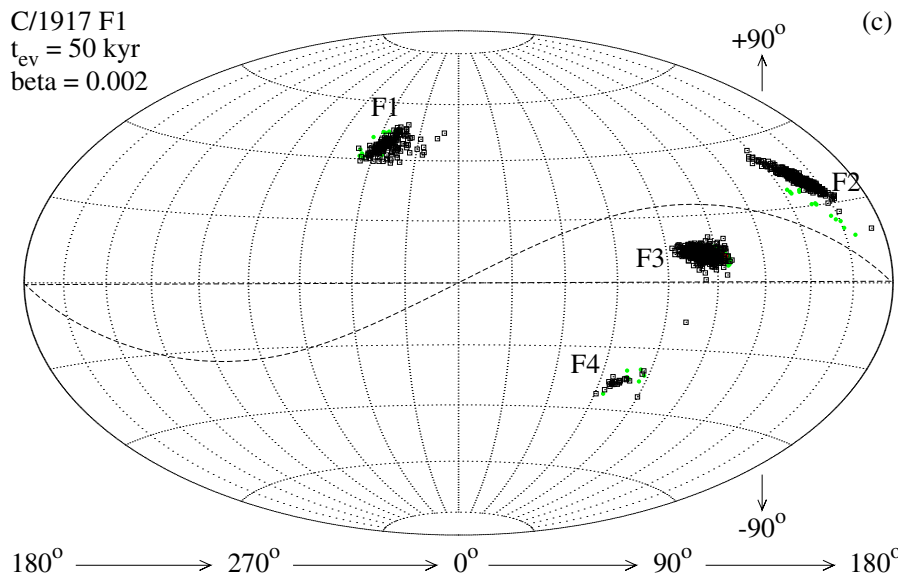

(c) $\mathrm{C} / 1917 \mathrm{~F} 1$

$\mathrm{t}_{\mathrm{ev}}=50 \mathrm{kyr}$

beta $=0.003$

$$
\begin{aligned}
& \mathrm{t}_{\mathrm{ev}}=50 \mathrm{kyr} \\
& \text { beta }=0.001
\end{aligned}
$$

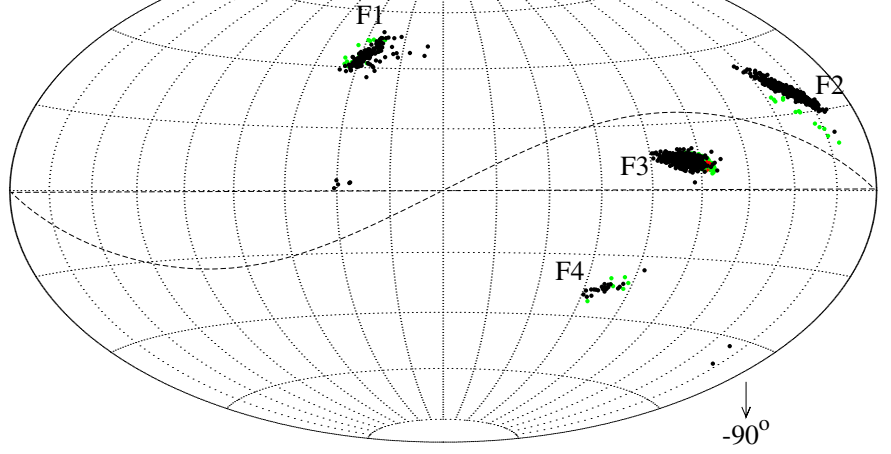

\section{C/1917 F1}

$\mathrm{t}_{\mathrm{ev}}=50 \mathrm{kyr}$

beta $=0.005$

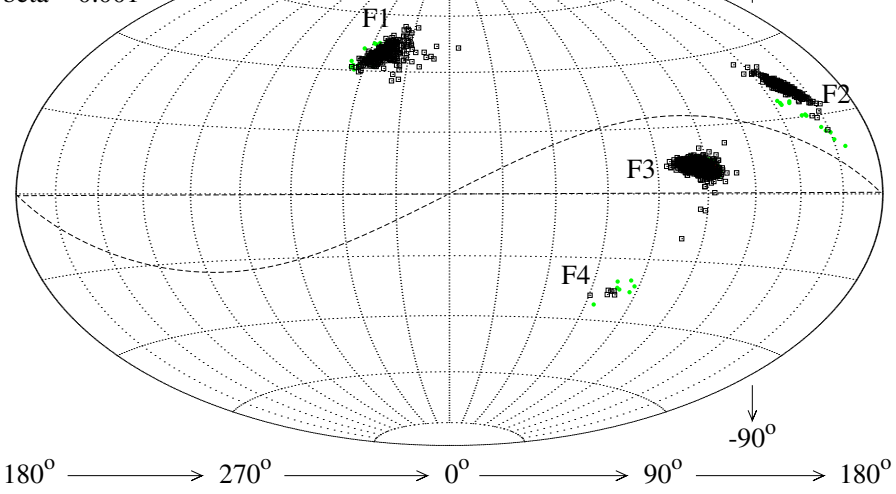

)

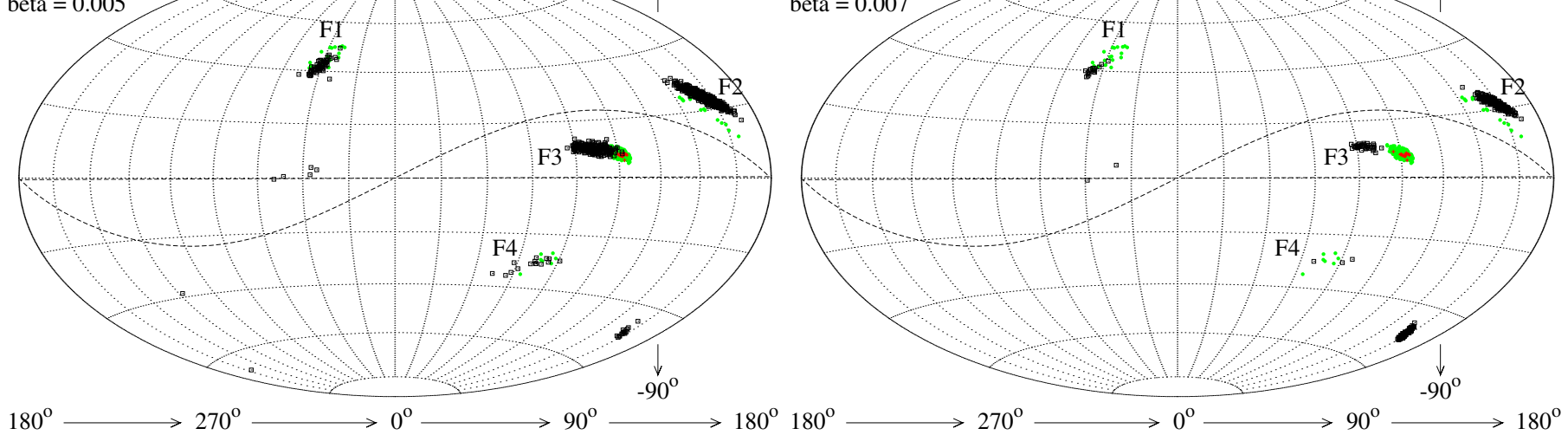

Fig. 3. Radiant positions of the modeled particles for different strength of the P-R drag. The positions of radiants are shown in the same way as those in Fig. 1a. The P-R drag influenced the motion of modeled particles in this case. The strength of the drag characterized with parameter $\beta$ was $0.0001,0.001,0.002,0.003,0.005$, and 0.007 at the streams with the radiants in plot a)-f), respectively. All the streams were modeled for time $50 \mathrm{kyr}$ in the past.

this filament consists of only a few TPs and completely disappears for a higher $\beta$ value.

\subsection{Streams modeled $20 \mathrm{kyr}$ before the present}

The F1 filament occurs, together with F3, for $t_{\mathrm{ev}}=20 \mathrm{kyr}$ and $\beta=0.0002$. If the higher value of $\beta=0.001$ is considered, F2 also occurs, but it is largely shifted northward (Fig. 2c). In addition, an unknown, small, compact filament with the radiant area near F3, but more southeastward and below the equator, occurs. Its mean argument of perihelion, longitude of ascending node, and inclination are $\omega=124.5^{\circ}, \Omega=80.4^{\circ}$, and $i=35.2^{\circ}$, respectively. These elements are similar to their DecemberMonocerotid counterparts (cf. $\omega=126.7^{\circ}, \Omega=80.1^{\circ}$, and $i=35.9^{\circ}$ ). However, the mean perihelion distance, radiant position, and geocentric velocity are different from those of the densest core of the December Monocerotids (cf. $q=0.217 \mathrm{AU}$, $\alpha=127.6^{\circ}, \delta=-5.1^{\circ}$, and $V_{g}=33.5 \mathrm{~km} \mathrm{~s}^{-1}$ and $q=0.189 \mathrm{AU}$, $\alpha=102.0^{\circ}, \delta=7.8^{\circ}$, and $V_{g}=41.9 \mathrm{~km} \mathrm{~s}^{-1}$; so while the first 
radiant area is situated below, the second radiant area is situated above the ecliptic). This filament is not indicated in the databases of the observed meteors. For $\beta=0.005$ and 0.01 , all TPs are deflected from the vicinity of the Earth's orbit and, thus, no filament occurs.

\subsection{Streams modeled $30 \mathrm{kyr}$ before the present}

The redundant filament, mentioned in the previous section, does not occur for $t_{\mathrm{ev}}=30 \mathrm{kyr}$. Otherwise, the result of the simulations for this $t_{\mathrm{ev}}$ is similar to that for $t_{\mathrm{ev}}=20 \mathrm{kyr}$. For the stream influenced with the P-R drag characterized with $\beta=0.001$, the distribution of radiants can be seen in Fig. $2 \mathrm{~d}$.

\subsection{Streams modeled $50 \mathrm{kyr}$ before the present}

\subsection{1. $\beta=0.0001$}

Filament F4 starts to occur in the models constructed for $t_{\mathrm{ev}} \geq$ 50 kyr. Specifically, there are a few meteors of this filament in the case of $t_{\mathrm{ev}}=50 \mathrm{kyr}$ and $\beta=0.0001$ (Fig. 3a). In this model, filament F1 is, however, overabundant, and the main accumulation of filament F2 is shifted north-northeastward of the observed radiant area. The prediction does not match the observed area of F3 perfectly either.

\subsection{2. $\beta=0.001$, and 0.0014}

For $\beta=0.001$ (Fig. 3b), a slightly better agreement than for $\beta=0.0001$ can be seen between the predicted and observed radiant areas of all four filaments. The prediction considering $\beta=0.0014$ (not shown) is almost the same as for $\beta=0.001$, but the overabundance in F1 is not as high, and the agreement in the case of filament F2 is slightly better.

\subsection{3. $\beta=0.002$}

Although still far from perfect, the best prediction perhaps occurs for $t_{\mathrm{ev}}=50 \mathrm{kyr}$ and $\beta=0.002$ (Fig. 3c). The agreement between the theory and observation is quite good, practically sufficient, in the case of filaments F1 and F4. However, it is still not significantly better than for $\beta=0.0014$ for filaments F2 and F3.

\subsection{4. $\beta=0.003$ and 0.005}

For $\beta=0.003$ (Fig. 3d), the quality of the prediction is similar to one for the previous value of $\beta$, but filament F4 is richer and F3 is slightly more shifted westward. The agreement is then worse for even higher $\beta$ values. For $\beta=0.005$ (Fig. 3e), the westward shift of F3 is a little larger than for 0.003 , and F1 becomes less numerous in comparison with its observed counterpart. A certain improvement occurs for F2, but it is still not sufficient. The predicted radiant area of $\mathrm{F} 4$ practically matches its observed counterpart, but another small, compact filament occurs in $\alpha \sim 160^{\circ}$ and $\delta \sim-50^{\circ}$ (in the southeastward direction from $\mathrm{F} 4$ ).

\subsection{5. $\beta=0.007$ and 0.01}

For $\beta=0.007$ (Fig. 3f), filament F3 is not numerous enough, and its predicted radiant area is completely outside its observed counterpart (but in touch with it). F1 and F4 are also less numerous, and F2 is still displaced. The redundant "southern" filament becomes more numerous than for $\beta=0.005$. Finally, the stream is deflected from the collision course with the Earth, when even higher $\beta$ values are assumed. For $\beta=0.01$, there are only three TPs in the orbits passing the orbit of the Earth within $0.05 \mathrm{AU}$.

\subsection{Streams modeled $70 \mathrm{kyr}$ before the present}

\subsection{1. $\beta=0.0005$}

Time $t_{\mathrm{ev}}=70 \mathrm{kyr}$ seems to be too long. The match is not as good as for $t_{\mathrm{ev}}=50 \mathrm{kyr}$. Considering $\beta=0.0005$, filament F1 is much more dispersed than the observed radiant area. F3 is also largely dispersed. A southward excess of the radiant area occurs with some radiants even below the equator. F4 is quite well populated but apparently shifted westward. The predicted interval of the declination of radiant area of filament F2 agrees with the corresponding observed interval, but it is still situated a little to the side and is clearly less numerous than the observed shower.

\subsection{2. $\beta=0.001$ and 0.002}

For $\beta=0.001$, we obtain similar results to the previous case, but with another diffuse filament having its radiant area near the equator and south-southwestward of F1 (Fig. 2f). If $\beta$ is increased to the value of 0.002 , we obtain a better match for filament F1, but filament F2 is smaller and does not match as well.

\subsection{3. $\beta=0.004$}

Another increase in $\beta$, to 0.004 , brings some improvement in comparison to 0.002: filament F1 fits the observations better (though still not perfectly), F2 becomes highly abundant but still discernibly separated from its observed counterpart and F3 is situated only northward of the equator, as it should be (the large dispersion disappeared), but its radiant area is still large and in a position apart from its observed counterpart. The redundant filament in the south-southwestward of F1 is much less abundant than for lower $\beta$-values, but still discernible. In this case, the predicted radiant area of filament F4 matches the observed one quite well.

\subsection{Question on November Orionids}

Vereš et al. (2011) suggest that comet C/1917 F1 is also the parent body of another established meteor shower, the November Orionids (No. 250), the mean orbit of which is similar to that of the December Monocerotids. In our models, where we also considered the P-R drag, we repeatedly searched for the theoretical November Orionids. However, no indication of such a shower occurred in our improved modeling either, which accords with the conclusion already made in NH14 when the P-R drag was ignored.

\section{Cloning the nominal orbit}

As seen in Figs. 2 and 3, which were constructed to show some aspects of the results described in the previous section, the $\mathrm{P}-\mathrm{R}$ drag alone does not, unfortunately, improve the agreement between the theory and observation enough. The discrepancy can, however, also occur if the non-gravitational effects, like the rocket effect, affect the orbit of the parent comet. Since we considered its nominal orbit, the site of the release of meteoroid particles from the cometary nucleus could be different from the site (perihelion) considered at the modeling. 


\subsection{The cloning procedure}

In the following part of the simulations, we create the clones of the nominal orbit of C/1917 F1. To obtain the cloned orbits, we modify some nominal orbital elements slightly. Specifically, we vary the eccentricity $e$, the argument of perihelion $\omega$, the longitude of ascending node $\Omega$, and the inclination $i$. Because of computational limitations, it is impossible to consider a large number of clones. To reduce this number to a necessary minimum, we do not vary the perihelion distance, $q$, and the time of perihelion passage, $T$. Past studies of major meteoroid streams yielded a quite large dispersion of eccentricity and semi-major axis of the stream members, but a significantly smaller variation of perihelion distance, so a change in $q$ can be expected to be lower than those of the other orbital elements, so we ignore it. We do not expect any change in $T$ greater than a few days because of the observational constraint. During such a short time, the main perturbing planets, especially Jupiter, do not change their positions much; therefore, a shift in $T$ most likely does not change the planetary perturbations calculated with respect to $T$ of the nominal orbit much.

We consider three values of the varied elements. Except for the nominal value, the low incremented and decremented values are also considered as the elements of clones. Specifically, the increments or decrements are chosen to be $\Delta e=0.005, \Delta i=1.8^{\circ}$, and $\Delta \omega=\Delta \Omega=3.5^{\circ}$. Because of some preliminary experiments, indicating the cloned orbits with a smaller inclination as more appropriate, we do not consider the incremented value of inclination, but only the values of $i-2 \Delta i, i-\Delta i$, and $i$.

Considering the orbit of each clone as the parent body of its own meteoroid stream, we create the model, for $t_{\mathrm{ev}}=50 \mathrm{kyr}$ (a "standard model") with no P-R drag, for each such parent and follow its evolution until the present. Using the TPs, which are in orbits passing around the orbit of the Earth within $0.05 \mathrm{AU}$ at present, we again predict the properties of individual filaments and evaluate the agreement between the mean orbit of each of the four predicted filaments and the corresponding observed mean orbit. The evaluation was done with the help of the Southworth-Hawkins (1963) $D$-discriminant. The $D$ values are given in Table 1.

With respect to the observational aspect of two-core filament F3 (there are two break points; see Fig. 6 in NH14), we also consider two variants, F3a and F3b, of this filament. The agreement is, subsequently, evaluated for these variants separately. A general agreement is then given as the average, $\langle D\rangle_{a}$, of the $D$ values for all four filaments (variant F3a is here taken into account). In Table 1, the clones are arranged by increasing $\langle D\rangle_{a}$-value. We can see that there is a better match between the theory and observation for only four clones, those with the serial numbers $4,3,17$, and 8 .

\subsection{Models for the cloned orbits No. 4 and 18 and P-R drag}

The best agreement occurred in the case of the cloned orbit with serial number 4 (clone No. 4). Since it is still imperfect, we again consider the influence of the P-R drag. In more detail, we use the orbit of clone No. 4 to model the streams for $t_{\mathrm{ev}}=10,20$, and $50 \mathrm{kyr}$ and several values of $\operatorname{parameter} \beta$.

\subsubsection{Models from Clone No. 4 for $t_{\mathrm{ev}}=10 \mathrm{kyr}$}

No progress is achieved for $t_{\mathrm{ev}}=10 \mathrm{kyr}$. If we assume $\beta=0.001$, only filament F3 occurs. Increasing $\beta$ to 0.002 (Fig. 4a), there is a good match of F3, and filament F2 also occurs in the prediction. Its radiant area is, however, displaced from its observed
Table 1. Values of the Southworth-Hawkins $D$-discriminant, $D_{j}$, between the mean observed and modeled orbits, where $j$ denotes the four assumed filaments of the meteoroid stream of C/1917 F1.

\begin{tabular}{rccccccc}
\hline \hline No. & $D_{1}$ & $D_{2}$ & $D_{3 a}$ & $D_{3 b}$ & $D_{4}$ & $\langle D\rangle_{a}$ & $\langle D\rangle_{b}$ \\
\hline 4 & 0.138 & 0.253 & 0.206 & 0.150 & 0.174 & 0.193 & 0.179 \\
3 & 0.197 & 0.316 & 0.126 & 0.037 & 0.196 & 0.209 & 0.186 \\
17 & 0.172 & 0.366 & 0.179 & 0.086 & 0.138 & 0.213 & 0.190 \\
8 & 0.133 & 0.342 & 0.257 & 0.194 & 0.219 & 0.237 & 0.222 \\
\hline Nom. & 0.198 & 0.297 & 0.159 & 0.095 & 0.316 & 0.242 & 0.226 \\
\hline 18 & 0.214 & 0.369 & 0.242 & 0.184 & 0.260 & 0.271 & 0.257 \\
14 & 0.557 & 0.291 & 0.169 & 0.159 & 0.283 & 0.325 & 0.323 \\
13 & 0.119 & 0.303 & 0.129 & 0.068 & 9.999 & 2.638 & 2.623 \\
12 & 0.173 & 0.314 & 0.187 & 0.198 & 9.999 & 2.668 & 2.671 \\
6 & 0.219 & 0.236 & 0.234 & 0.116 & 9.999 & 2.672 & 2.643 \\
10 & 0.210 & 0.300 & 0.185 & 0.139 & 9.999 & 2.674 & 2.662 \\
7 & 0.092 & 0.399 & 0.212 & 0.120 & 9.999 & 2.675 & 2.652 \\
15 & 0.189 & 0.331 & 0.185 & 0.141 & 9.999 & 2.676 & 2.665 \\
16 & 0.228 & 0.324 & 0.212 & 0.155 & 9.999 & 2.691 & 2.676 \\
1 & 0.217 & 0.375 & 0.181 & 0.147 & 9.999 & 2.693 & 2.685 \\
11 & 0.218 & 0.344 & 0.215 & 0.150 & 9.999 & 2.694 & 2.678 \\
22 & 0.235 & 0.426 & 0.245 & 0.193 & 9.999 & 2.726 & 2.713 \\
2 & 0.262 & 0.276 & 0.401 & 0.349 & 9.999 & 2.734 & 2.722 \\
9 & 0.749 & 0.280 & 0.171 & 0.059 & 9.999 & 2.800 & 2.772 \\
19 & 0.198 & 9.999 & 0.132 & 0.091 & 9.999 & 5.082 & 5.072 \\
20 & 0.196 & 9.999 & 0.136 & 0.096 & 9.999 & 5.082 & 5.072 \\
21 & 0.201 & 9.999 & 0.158 & 0.104 & 9.999 & 5.089 & 5.076 \\
24 & 0.200 & 9.999 & 0.159 & 0.112 & 9.999 & 5.089 & 5.077 \\
23 & 0.201 & 9.999 & 0.173 & 0.114 & 9.999 & 5.093 & 5.078 \\
5 & 9.999 & 0.375 & 0.136 & 0.118 & 9.999 & 5.127 & 5.123 \\
\hline & & & & & & &
\end{tabular}

Notes. $\langle D\rangle_{a},\langle D\rangle_{b}$ are the averaged values $\left(D_{1}+D_{2}+D_{3 a}+D_{4}\right) / 4$ and $\left(D_{1}+D_{2}+D_{3 b}+D_{4}\right) / 4$; "No." refers to the serial number of the clone; "Nom." indicates the nominal orbit. The data are arranged in increasing order of average discriminant $\langle D\rangle_{a}$. (An ad hoc value of 9.999 is supplied for $D_{j}$ in case the clone did not produce the particular filament.)

counterpart. No F1 or F4 is present. For $\beta=0.003$, filament F3 is almost "extinct". F2 becomes quite numerous, but still separated from the observed radiant area. F1 and F4 are still missing. For higher $\beta$-values, the prediction fails even more. Specifically, only F2, with the radiant area aside the observed one, is predicted for $\beta=0.005$. Then, this filament becomes more compact and less numerous for $\beta=0.007$. Finally, for $\beta=0.009$, filaments F1 to F4 disappear completely. Instead of these, a new, redundant, compact filament in $\alpha \sim 150^{\circ}$ and $\delta \sim-40^{\circ}$ is predicted.

\subsubsection{Models from Clone No. 4 for $t_{\mathrm{ev}}=20 \mathrm{kyr}$}

In the models for $t_{\mathrm{ev}}=20 \mathrm{kyr}$, we assume a sequence of $\beta$ values ranging from 0.001 to 0.009 with the step of 0.002 and $\beta=0.013$. Filament 4 is not predicted in any of these models. However, we achieved a partial progress in predicting the most problematic, likely, filament F2. A good agreement between its predicted and observed radiant areas is obtained for $\beta=0.009$ (Fig. 4b), as well as 0.007 .

Unfortunately, there is a poor match of filaments F1 and F3. F1 is not numerous enough, and the radiant area of F3 is shifted westward. In addition, a redundant shower with a strip-like radiant area having the mean $\alpha \sim 160^{\circ}$, and $\delta<-50^{\circ}$ is predicted in all models for $t_{\mathrm{ev}}=20 \mathrm{kyr}$.

\subsubsection{Models from Clone No. 4 for $t_{\mathrm{ev}}=50 \mathrm{kyr}$}

Models based on the cloned orbit No. 4 for $t_{\mathrm{ev}}=50 \mathrm{kyr}$ provide a prediction of all four filaments, but the prediction of hardly predictable filament F2 is always worse than for $t_{\mathrm{ev}}=20 \mathrm{kyr}$ 


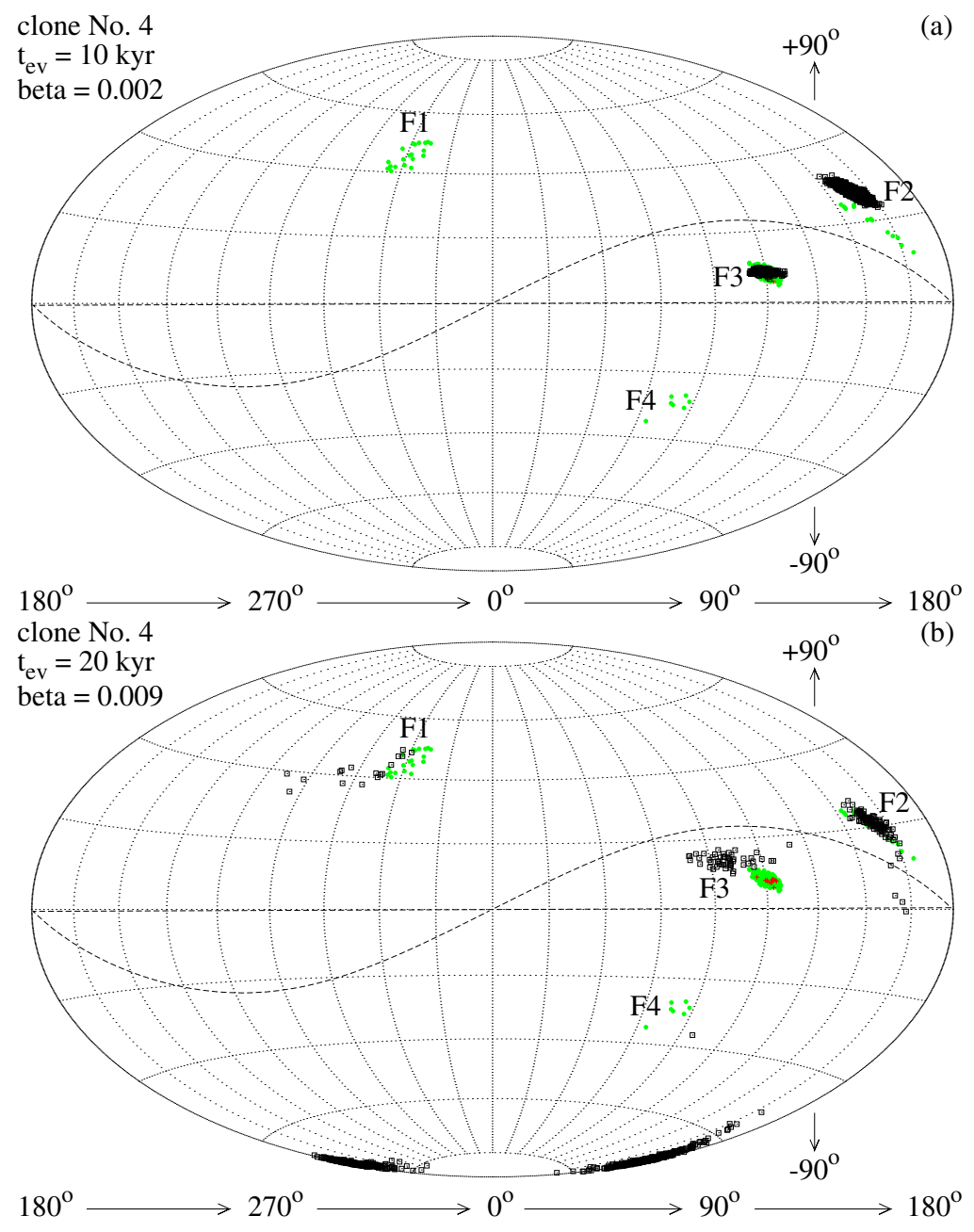

Fig. 4. Radiant positions of the particles modeled using a cloned orbit of the comet. The positions of radiants are shown in the same way as those in Fig. 1a. However, the initial orbit to model the stream was the cloned orbit No. 4 and P-R drag influenced the motion of modeled particles in this case. In plot a), the stream was modeled in time $10 \mathrm{kyr}$ before the present and parameter $\beta=0.002$. In plot $\mathbf{b})$, the stream was modeled in time 20 kyr before the present and $\beta=0.009$.

and $\beta=0.009$. Specifically, we considered the values $\beta=0.001$, 0.005 , and 0.009 for $t_{\mathrm{ev}}=50 \mathrm{kyr}$. For $\beta=0.001$, modeled filaments F1 and F3 are shifted toward higher right ascension; F2 is shifted, a little, toward lower right ascension and higher declination; and F4 consists of only two TPs. There are quite a large number of TPs in the region with the declination $\delta<-60^{\circ}$.

For $\beta=0.005$, we can state a little better match of F1 and F3 with observation than in the previous case. However, the match is worse for F2. The radiant area of filament F4 is, here, clearly separated from its observed counterpart. It is shifted toward a lower declination. And, there is even a higher concentration of the TPs in region $\delta<-70^{\circ}$.

The prediction failed for $\beta=0.009$. Only a few TPs represent each of the four filaments. Again, a high concentration of TPs in region $\delta<-70^{\circ}$ is predicted.

\subsubsection{Models from Clone No. 18}

Seeing most of the results described above, it seems that the largest problem is to achieve a good match for filament F2. The $\mathrm{P}-\mathrm{R}$ drag is able to reduce the declination of radiant of F2 members, but the right ascension typically remains too large. In the next step, we try to model the stream using the cloned orbit No. 18 with one of the lowest values of this parameter in the "standard stream". Some cloned orbits yielded an even smaller right ascension, but their "standard stream" did not consist of all four observed filaments, in contrast to the cloned orbit No. 18.

Starting from the latter, we simulated several streams for $t_{\mathrm{ev}}=10,20$, and $50 \mathrm{kyr}$ and a few values of parameter $\beta$.
Unfortunately, the attempt to progress with the help of this clone completely failed. No good match was achieved, even in the case of only a single filament. Concerning filament F2, the difference between the predicted and the observed mean inclination of the radiant area appeared to be larger than in the case of nominal orbit.

\section{Search for a convergence of the orbits of real meteors}

\subsection{The principle and method}

If a swarm of particles is released from the surface of a parent body, they start orbiting the Sun along very similar orbits. With elapsing time, this similarity decreases, and the orbits become more and more dispersed. If we knew the exact orbits of a set of meteoroids from a stream, we could reconstruct their evolution by integrating these orbits back in time. In such a backward integration, the dispersion of orbits should decrease to reach the original state immediately after the particle ejection.

In reality, we only know the meteoroid orbits with a certain precision. The uncertainty in their determination means that their backward integration does not result in a smaller, but rather in a gradually larger dispersion. As a result, we usually cannot reveal the actual orbit of the parent body in the time of meteoroid production on the basis of the known orbits of these meteoroids.

Nevertheless, if we know a relatively large number of the orbits of meteoroids in a given stream, there is still a chance that their backward integration will reveal a convergence of at least 


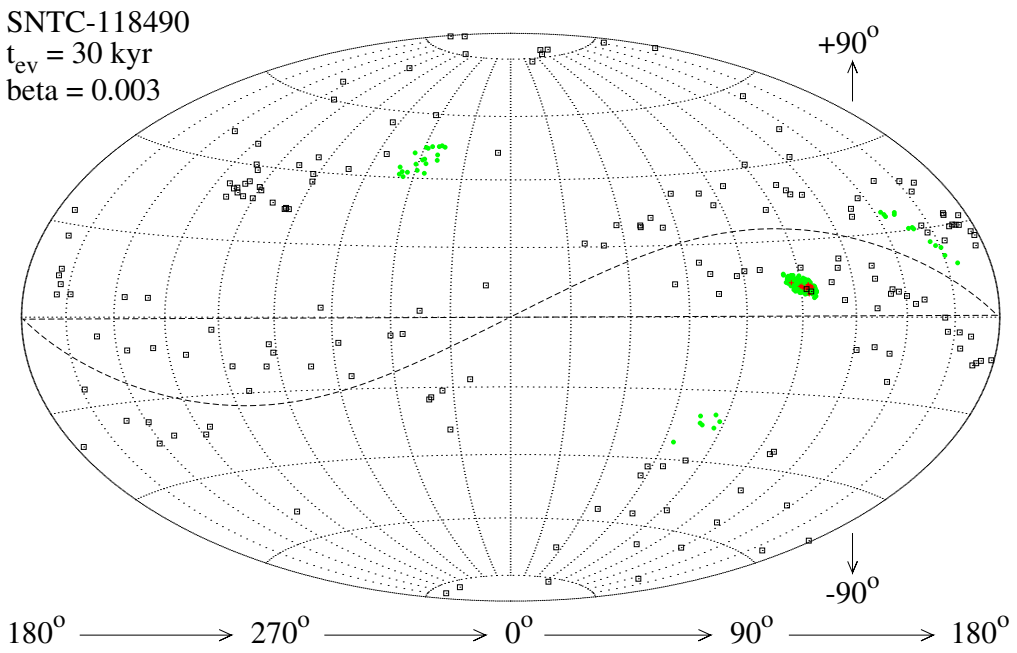

Fig. 5. Radiant positions of the particles modeled using an orbit obtained by backward integration of observed meteors. The positions of radiants are shown in the same way as those in Fig. 1a. However, the initial orbit to model the stream was that of video meteor SNTC-118490, which was obtained via numerical integration of the current orbit for time $30 \mathrm{kyr}$ before the present in this case. The P-R drag influencing the motion of modeled particles characterized with $\beta=0.003$ was considered, too. a fraction of them. Such a convergence could perhaps indicate the parameters of the orbit of the parent in the time of meteoroid production. Considering this possibility, we integrate the sets of known orbits from the photographic and video databases used, which correspond to the filaments F1-F4 of the C/1917 F1 stream, and search to see if an orbit from one filament became similar in 10, 20, 30, or $50 \mathrm{kyr}$ in the past to an orbit (or orbits) from another filament (or filaments). Expressed in more detail, we perform several backward integrations, each for another P-R parameter $\beta$. Values of $\beta$ equal to $0,0.0005,0.001,0.005,0.009$, and 0.013 are considered for $t_{\mathrm{ev}}=10,20$, and $30 \mathrm{kyr}$. With respect to the results obtained in these simulations, we considered values $\beta=0.00001,0.003,0.005$, and 0.007 for $t_{\mathrm{ev}}=50 \mathrm{kyr}$.

The similarity of the orbits from different filaments in the given past is evaluated with the help of the Southworth-Hawkins (1963) $D$-discriminant. A given pair of orbits was regarded as similar if $D<0.2$.

\subsection{Implied convergence}

For $t_{\mathrm{ev}}=50 \mathrm{kyr}$, no single filament currently crossing the Earth's orbit was predicted. For $\beta=0.007$, the aphelion of the parent meteoroid has increased above 1000 AU so far, therefore any observable meteoroid stream could be expected, so we did not create any model in this case.

A certain improvement could be stated for $t_{\mathrm{ev}}=10$ and $20 \mathrm{kyr}$. At any rate, the best convergence occurred for $\beta=0.005$ in time $30 \mathrm{kyr}$ before the present. In this model, the orbit of video meteor No. 118490 from filament F1 became similar, in $30 \mathrm{kyr}$, to that of video meteor No. 49303 from filament $F 2$. The $D$ value between the orbits of this pair was 0.140 . At the same time, the orbit of No. 118490 from F1 became similar to that of video meteor No. 112248 from filament F3. This time, $D=0.099$. The sum of these $D$ values was the lowest of all found similarities and equal to 0.239 . The elements of these similar orbits are given in Table 2.

Considering the orbits of meteors from three filaments, which were similar $30 \mathrm{kyr}$ ago, we can suppose that (i) the meteors were released from the parent around this time; and (ii) the orbit of the parent body was very similar to the orbits of these three meteors at that time. As a result, we approximatively identify the orbit of the parent to that of meteor No. 118490. In other words, we assumed that the orbit of C/1917 F1 had the elements given in the first line in Table 2 in a time of about $30 \mathrm{kyr}$ in the past.
Table 2. Largest convergence of orbits of real meteors found from three filaments (fils. F1 to F3) of the C/1917 F1 stream.

\begin{tabular}{rrrccccc}
\hline \hline fil. & IC & $\begin{array}{c}q \\
{[\mathrm{AU}]}\end{array}$ & $\begin{array}{c}e \\
{[1]}\end{array}$ & $\begin{array}{c}\omega \\
{[\mathrm{deg}]}\end{array}$ & $\begin{array}{c}\Omega \\
{[\mathrm{deg}]}\end{array}$ & $\begin{array}{c}i \\
{[\mathrm{deg}]}\end{array}$ & $D$ \\
\hline F1 & 118490 & 0.313 & 0.993 & 34.3 & 215.5 & 56.7 & \\
F2 & 49303 & 0.442 & 0.977 & 32.7 & 214.9 & 58.8 & 0.140 \\
\hline F1 & 118490 & 0.313 & 0.993 & 34.3 & 215.5 & 56.7 & \\
F3 & 112248 & 0.317 & 0.988 & 41.1 & 212.6 & 56.8 & 0.099 \\
\hline
\end{tabular}

Notes. IC is the identification code of a meteor in the SonotaCo video database; $q, e, \omega, \Omega$, and $i$ are the orbital elements from September 26.5, 28005 B.C., when the similarity occurred.

\subsection{Stream modeling and prediction of filaments}

Using the orbit mentioned at the end of the previous subsection, we integrated the position of meteor No. 118490 to the perihelion passed in the time closest to $30 \mathrm{kyr}$ and then modeled the theoretical stream. Subsequently, we followed the evolution of stream particles up to the present and predicted the mean dynamical properties of filaments F1 to F4.

In the first model of this sequence, we assumed $\beta=0.005$. At the end, it was found that the radiants of meteors approaching the Earth's orbit were dispersed over the whole sky. This was the effect of an ejection speed that was too high, which was implicitly assumed. We note that, in the procedure used, the ejection speed of each TP from the parent surface is assumed to be equal to $0.001 v_{\mathrm{p}}$, where $v_{\mathrm{p}}$ is the heliocentric velocity of the parent in its perihelion. However, in this case, the parent body occurred in the orbit with an extremely small perihelion distance ( $q=0.01586 \mathrm{AU}) 30 \mathrm{kyr}$ ago. Such a small perihelion distance implied the high ejection speed of $333 \mathrm{~m} \mathrm{~s}^{-1}$, so we performed a new modeling with the ejection speed reduced by a factor of four. At the same time, we assumed a lower strength of the P-R drag with $\beta=0.003$.

It appears that the video meteor No. 118490 moved in a very special orbit in the context of parent body of a meteoroid stream. Reducing the ejection velocity had practically no effect. Also in this simulation, the TPs approached the Earth's orbit from almost all directions (see Fig. 5). The hypothetical parent body considered here is an example of such a parent, which supplies the particles immediately to the sporadic background, without the stage of a compact stream. This type of stream is, however, beyond the scope of our current study. 


\section{Conclusion}

Our attempt to improve the prediction of the properties of meteor showers associated with comet C/1917 F1 by modeling its stream and considering the P-R drag, as well as nongravitational effects, only resulted in partial success. When the nominal orbit of the comet is considered, the P-R drag clearly helps to improve the prediction in the case of filaments F1, F3, and F4. The prediction for F2 is also better than that without the $\mathrm{P}-\mathrm{R}$ drag, but far from perfect.

We found a cloned orbit, which resulted in a practically perfect prediction of the properties of problematic filament F2. This orbit could be the actual orbit of C/1917 F1 in the appropriate past period owing to unknown non-gravitational effects. Unfortunately, the simultaneous perfect match of the other three filaments does not occur in the modeling concerned. In reality, no explanation for this specific case can be made.

Our study illustrates the different ages of particles in various filaments, i.e. the different ages of meteoroids in the various showers originating in the same parent body. While the members of December Monocerotids corresponding to filament F3 can be observed soon after their release from the parent comet, most probably much earlier than after $5 \mathrm{kyr}$, the April $\rho$-Cygnids corresponding to filament F1 can occur in the vicinity of Earth's orbit and collide with this planet not earlier than about $20 \mathrm{kyr}$. According to our simulations, their typical age is at least about another $10 \mathrm{kyr}$ longer. The particles belonging to filament F4, which correspond to a still unknown shower, can collide with the Earth only after about 50 kyr. Maybe the meteoroids released from C/1917 F1 can hardly survive such a long time in interplanetary space, and this could be the reason the real counterpart of filament F4 is observed to be relatively less numerous.

We have to admit that we investigated only a relatively small set of a huge number of possible combinations of modified orbits, each expanded into an even larger number when a variety of strengths of the P-R drag is assumed. A much more robust modeling could possibly reveal a convergence in cloning at a narrow interval of P-R drag strength in the future. If such a success was achieved, the studies of meteoroid streams could become a tool for recovering the history of the orbital evolution of their parent bodies owing to the action of non-gravitational effects.

Acknowledgements. J. Vaubaillon is grateful to the CINES staff for the use of the occigen machine on which heavy calculations were performed. L. Neslušan and M. Hajduková acknowledge the support from the Project ITMS No. 26220120009, based on the supporting operational Research and development program financed by the European Regional Development Fund. Their work was also supported by the Slovak Research and Development Agency under contract No. APVV-0517-12 (M.H.) and by the VEGA - the Slovak Grant Agency for Science, grant No. 2/0031/14 (L.N.).

\section{References}

Asklöf, S. 1932, Arkiv for Mat. Astron. och Fys., 23A, No. 11

Drummond, J. 1981, Icarus, 45, 545

Hajduková, M. Jr., Rudawska, R., Kornoš, L., \& Tóth, J. 2015, Planet. Space Sci., 118, 28

Hawkins, G. S. 1963, Smithsonian Contrib. Astrophys., 7, 53

Jopek, T. J., \& Kaňuchová, Z. 2014, in Meteoroids 2013, Proc. Internat. Conf. held in Poznań, Poland, August 26-30, eds. T. J. Jopek, F. J. M. Rietmeijer, J. Watanabe, \& I. P. Williams (Poznań: A. M. University Press), 353

Kornoš, L., Koukal, J., Piffl, R. \& Tóth, J. 2014, in Proc. International Meteor Conference, held in Poznań, Poland, eds. M. Gyssens, P. Roggemans, \& P. Zoladek (International Meteor Organization), 23

Kresáková, M. 1974, Bull. Astron. Inst. Czechosl., 9, 88

Lindblad, B. A. 1971, Space Res., 11, 287

McCrosky, R. E., \& Posen, A. 1961, Smithsonian Contr. Astrophys., 4, 15

Neslušan, L., \& Hajduková, Jr. 2014, A\&A, 566, A33

Neslušan, L., Svoreň, J., \& Porubčan, V. 1995, Earth, Moon, Planets, 68, 427

Neslušan, L., Svoreň, J., \& Porubčan, V. 2013, Earth, Moon, Planets, 110, 41

Neslušan, L., Porubčan, V., \& Svoreň, J. 2014, Earth, Moon, Planets, 111, 105

Olsson-Steel, D. 1987, Austr. J. Astron., 2, 21

Sekanina, Z., \& Southworth, R. B. 1975, Physical and dynamical studies of meteors. Meteor-fragmentation and stream-distribution studies, Final Report (Cambridge, MA: Smithsonian Astrophys. Obs.)

SonotaCo. 2009, WGN - Journal of the IMO, 37, 55, http://sonotaco.jp/ doc/SNM/

Southworth, R. B., \& Hawkins, G. S. 1963, Smithsonian Contrib. Astrophys., 7, 261

Tomko, D., \& Neslušan, L. 2012, Earth, Moon, Planets, 108, 123

Vereš, P., Kornoš, L., \& Tóth, J. 2011, MNRAS, 412, 511 